\title{
Simulación Numérica de la Propagación de una Fisura en un Material Degradado por efecto de la Fragilización por Hidrógeno
}

Jorge A. Palma ${ }^{1}$, José M.A. Barbosa ${ }^{2}$ y Antonio A. Silva ${ }^{3}$

(1) Universidad Federal de Campina Grande, Postgrado en Ciencias e Ingeniería de Materiales, Av. Aprígio Veloso, 882 - Bodocongó, C. Grande, PB - Brasil (e-mail: jorge_palma_c@yahoo.com.br) (2) Universidad Federal de Pernambuco, Dpto. de Ing. Mecánica, Av. Hélio Ramos, s/n, Ciudad Universitaria, Recife, PE - Brasil (e-mail: jmab@ufpe.br)

(3) Universidad Federal de Campina Grande, Dpto. de Ing. Mecánica, Av. Aprígio Veloso, 882 Bodocongó, C. Grande, PB-Brasil (e-mail: almeida@dem.ufcg.edu.br)

\begin{abstract}
Resumen
Se presenta una formulación numérica basada en una combinación de la mecánica de la fractura y de la mecánica del daño continuo para tratar el problema de la propagación de una fisura en régimen elástico, bajo el efecto de una acción mecánica definida por una carga estática aplicada en el modo de fractura I y de una acción ambiental caracterizada por la difusión de hidrógeno a través de la estructura cristalina del material. Las ecuaciones que describen la evolución de las variables en la punta de la fisura forman un sistema no lineal de ecuaciones diferenciales ordinarias que es resuelto con el método de Runge-Kutta de $4^{\circ}$ orden. Los resultados obtenidos muestran la influencia del hidrógeno en la disminución del tiempo de inicio y propagación de fisuras, lo que es consistente con las observaciones macroscópicas del fenómeno de fragilización por hidrógeno
\end{abstract}

Palabras clave: mecánica de la fractura, simulación numérica, propagación de fisuras, fragilización por hidrógeno

\section{Numerical Simulation of Crack Propagation in a Degraded Material under Hydrogen Embrittlement Effects}

\begin{abstract}
A numeric formulation is presented based on a combination of fracture mechanics and continuum damage mechanics to treat the problem of the crack propagation in elastic regime, under the effect of a mechanical action defined by a static load applied in the tensile mode and of an environmental action characterized by hydrogen diffusion into the lattice. The equations that describe the evolution of the variables at the crack tip form a non-linear system of ordinary differential equations that is solved using the $4^{\text {th }}$ order Runge-Kutta method. The results show the influence of hydrogen on the decrease of the time at which cracks starts and propagates, which is consistent with macroscopic observations of the hydrogen embrittlement phenomenon.
\end{abstract}

Keywords: fracture mechanics, numerical simulation, cracks propagation, hydrogen embrittlement 


\section{INTRODUCCIÓN}

La respuesta no lineal de los sólidos, observada en la macro-escala, es una manifestación de procesos irreversibles que ocurren en su micro-estructura y que pueden originarse a partir de microdefectos que constituyen el daño inicial del material. Dependiendo de las condiciones ambientales y debido a la existencia de solicitaciones mecánicas estáticas o cíclicas, aún que la respuesta global del sistema permanezca dentro de los límites del régimen elástico, ese daño inicial puede evolucionar, llevando a la formación y crecimiento de micro-fisuras, siendo frecuente que factores ambientales como el aire húmedo, el agua del mar y otros medios activos intensifiquen su inicio y propagación (Villalba y Atrens, 2009). Esos procesos están relacionados con fenómenos que muestran gran influencia en la disminución de la resistencia de los materiales a la fatiga y fractura

Un fenómeno típico es la fragilización por hidrógeno, que cambia las propiedades mecánicas de los metales y aleaciones metálicas, lo que resulta en la disminución de su resistencia a la fractura y en la aceleración del crecimiento de fisuras (Hertzberg, 1996; Bolotin, 1999; Gangloff, 2003; Gangloff, 2005; Yongwon y Gangloff, 2007; Zhang et al., 2007), siendo definida como el daño ocasionado por la presencia del hidrógeno atómico en la estructura cristalina del material bajo tensiones residuales o aplicadas (Stroe, 2006; Louthan, 2008). El hidrógeno libre y el químicamente asociado pueden ser fuentes de la degradación de las propiedades de los materiales al provocar fallas estructurales relacionadas con procesos resultantes de su difusión a través del reticulado cristalino, tales como la nucleación de vacíos y la formación de burbujas, que pueden ser calificados como procesos de micro-damnificación y, desde un punto de vista fenomenológico, descritos como el daño producido por acciones puramente mecánicas. Aquí, el término fragilización por hidrógeno cubre los mecanismos de damnificación asociados con la difusión del hidrógeno en los metales.

Después del primer modelo propuesto por Kachanov en 1958, fue desarrollada una impresionante actividad en investigaciones destinadas al desarrollo de modelos viables para la descripción del daño en una gran cantidad de materiales, como el acero (Celentano y Chaboche, 2007), materiales compuestos (Maimi, 2006) y otros, con el propósito de resolver una amplia gama de problemas conectados con la degradación de las propiedades de los materiales, tales como efectos térmicos e inerciales (Barbosa, 1998) o factores ambientales (Duda et al., 2007a). Recientemente, Duda et al. (2007b), formularon un modelo unidimensional en el contexto de la mecánica de los medios continuos para simular deformación, degradación y difusión del hidrógeno en sólidos elásticos. Palma Carrasco (2008), simuló el crecimiento de una fisura en un cuerpo material de acero de alta resistencia sometido a efectos mecánicos y ambientales, mediante el acoplamiento de modelos numéricos de las mecánicas de la fractura elástica lineal y del daño continuo.

En este artículo se presentará el modelo de inicio y crecimiento de fisuras en condiciones de asistencia por el ambiente propuesto por Bolotin y Shipkov (2001a), utilizado en el análisis numérico de problemas relacionados con la corrosión bajo tensión (SCC) y la fatiga con corrosión (CF), para simular numéricamente el crecimiento y propagación, en régimen elástico, de una fisura en una placa metálica plana sometida a una carga mecánica estática en el modo de fractura I y al efecto de una acción ambiental no corrosiva, definida por la presencia de hidrógeno atómico en la estructura cristalina del material. Ambas acciones en conjunto caracterizan el fenómeno de fragilización por hidrógeno.

\section{MODELO DE INICIO Y CRECIMENTO DE FISURAS BAJO ASISTENCIA DEL AMBIENTE}

Este modelo, basado en una síntesis de la Mecánica del Daño Continuo y de la Mecánica de la Fractura, considera que la propagación de la fisura es el resultado de la interacción entre las condiciones de estabilidad del cuerpo fisurado como un sistema mecánico y el proceso de acumulación de daño. El modelo incluye ecuaciones cinéticas para la acumulación de cada tipo de daño y ecuaciones que describen la evolución de la punta de la fisura y la influencia del proceso de acumulación de daño sobre las fuerzas generalizadas de resistencia a la fractura. Adicionalmente, incorpora una ecuación de transferencia de masa y aborda una teoría general para la propagación de fisuras desarrollada por Bolotin (1999). 
Este acoplamiento, asociado a las condiciones de equilibrio, estabilidad y propagación de fisuras, posibilita la modelización del crecimiento de una fisura y de la fractura final provocados por una carga estática y el efecto de un fenómeno ambiental, como la fragilización por hidrógeno.

\section{Daño asistido por el hidrógeno}

Para modelizar el proceso de acumulación de daño en condiciones de asistencia por el hidrógeno, Bolotin (1999) y Bolotin y Shipkov (2001a; 2001b) utilizan el modelo escalar de daño más simple de Kachanov y Rabotnov, que caracteriza el daño disperso a través de un campo escalar $\omega=(x, t)$, e introducen una medida especial para cada tipo de daño y la correspondiente ecuación cinética que gobierna su evolución en el tiempo. Aún cuando únicamente son consideradas acciones mecánicas, realizan una diferenciación entre el daño provocado por cargas estáticas y el debido a otras solicitaciones mecánicas, como las cíclicas; el daño provocado por la difusión de un agente ambiental a través de la estructura cristalina del material también es diferenciado del daño producido por un proceso de corrosión. Por lo tanto, el campo de daño introducido simbólicamente por $\omega=(x, t)$, que continúa variando en el intervalo $[0,1]$ (igual a cero cuando no existe daño e igual a 1 cuando el material se encuentra completamente damnificado), pasa a ser representado por un conjunto de campos escalares de daño, $\omega_{1}(x, t), \ldots, \omega_{n}(x, t)$.

El modelo asume que las ecuaciones cinéticas para las medidas particulares del daño son ecuaciones diferenciales de $1^{\text {er }}$ orden. El daño producido por una carga estática, $\omega_{s}$, en el modo de fractura I, es dado por:

$$
\frac{\partial \omega_{s}}{\partial t}=\frac{1}{t_{c}}\left(\frac{\sigma-\sigma_{\text {th }}}{\sigma_{s}}\right)^{m_{s}}
$$

donde $\sigma$ es el valor medio de la tensión de tracción que actúa en un punto material considerado. $\sigma_{\mathrm{s}}$ caracteriza la resistencia al daño producido por la carga estática y $\sigma_{\text {th }}$ es un parámetro de resistencia umbral. El exponente $m_{s}$ es similar a los exponentes de las ecuaciones de las curvas de fatiga y de la tasa de crecimiento de la fisura que, bajo ciertas condiciones, tiene valores específicos (Bolotin, $1999) ; t_{c}$ es una constante de tiempo cuya magnitud puede depender del parámetro $\sigma_{\mathrm{s}}$ o ser escogida de forma arbitraria.

El daño por fragilización, $\omega_{h}$, como un componente aditivo de la medida del daño total, que solamente depende de la concentración del hidrógeno en la punta de la fisura es representado por:

$\omega_{h}(x, t)=\Psi_{h}(t)\left(1-\frac{\xi}{\lambda_{h}}\right)$

donde $\lambda_{\mathrm{h}}$ es la longitud de la zona de proceso de la fragilización y $\psi_{\mathrm{h}}(\mathrm{t})$ es el daño en la punta de la fisura provocado por el hidrógeno. $\xi=\mathrm{x}-\mathrm{a}, 0 \leq \xi \leq \lambda_{\mathrm{h}}$. Para $\xi>\lambda_{\mathrm{h}}, \omega_{\mathrm{h}} \equiv 0$.

La evolución del daño disperso representado en (1) y (2), en la punta de la fisura, puede ser expresada por:

$$
\begin{aligned}
& \frac{d \psi_{s}}{d t}=\frac{1}{t_{c}}\left(\frac{\sigma-\sigma_{t h}}{\sigma_{s}}\right)^{m_{s}} \\
& \frac{d \Psi_{h}}{d t}=\frac{\Psi_{h}^{\infty}}{c_{h}} \exp \left(-\frac{c_{t}}{c_{h}}\right)
\end{aligned}
$$


donde $\Psi_{\mathrm{h}}^{\infty}$ es la medida del daño en la punta de la fisura correspondiente a un estado de saturación; $c_{t}$ es la concentración en la punta de la fisura y $c_{h}$ es la concentración estacionaria del hidrógeno, alcanzada para una fisura estacionaria.

La concentración en la punta de la fisura está relacionada con su radio de curvatura, $\rho$, cuya evolución está gobernada por varios procesos, tales como el crecimiento de la fisura y la acumulación de los daños. La ecuación diferencial para la evolución del radio efectivo de la punta de una fisura plana (con dimensión a) está dada por:

$$
\frac{d \rho}{d t}=\frac{\rho_{s}-\rho}{\lambda_{a}} \frac{d a}{d t}+\left(\rho_{b}-\rho\right) \frac{d\left(\psi_{s}+\psi_{h}\right)}{d t}
$$

El primer término del lado derecho de la ecuación describe el aguzamiento de la punta debido al crecimiento de la fisura con la tasa da/dt hasta la magnitud $\rho_{\mathrm{s}} ; \lambda_{\mathrm{a}}$ caracteriza la distancia que su punta debe recorrer para que el efecto de aguzamiento sea considerado representativo. El segundo término describe su embotamiento debido a la acumulación del daño mecánico y del daño debido al hidrógeno hasta la magnitud $\rho_{\mathrm{b}} . \Psi_{\mathrm{s}}$ y $\Psi_{\mathrm{h}}$ son las magnitudes de $\omega_{\mathrm{s}}$ y $\omega_{\mathrm{h}}$ en la punta de la fisura.

La última relación que cierra el conjunto de ecuaciones que gobiernan el modelo, interrelaciona las fuerzas generalizadas de resistencia con las medidas del daño al frente de la punta de la fisura. El trabajo específico de fractura es dado por:

$Y=Y_{0}\left[1-x\left(\omega_{\sigma}+\omega_{h}\right)^{\alpha}\right]$

donde $\mathrm{Y}_{0}$ es el trabajo específico de fractura para un cuerpo no damnificado, $\mathrm{X}$ caracteriza la tenacidad a la fractura residual para el material damnificado y a es un parámetro material. Por lo tanto, $\mathrm{\gamma}=\mathrm{y}_{0}(1-\mathrm{x})$ caracteriza el trabajo específico de fractura residual para un material completamente damnificado.

A partir de la Ecuación (6) y considerando $\Gamma_{0} \equiv \gamma_{0}$, para $\alpha>0$ y $x \geq 1$ en procesos de fragilización por hidrógeno, se puede obtener la fuerza generalizada de resistencia para una fisura plana, dada por:

$\Gamma=\Gamma_{0}\left[1-\chi\left(\Psi_{\mathrm{s}}+\Psi_{\mathrm{h}}\right)^{\alpha}\right]$

\section{Crecimiento de fisuras}

Un cuerpo fisurado bajo la acción de una carga y los efectos del ambiente es un tipo especial de sistema mecánico, cuyo estado corriente es descrito por un grupo de coordenadas-L (Lagrangianas), que describen el campo de desplazamientos en el cuerpo y otro de coordenadas-G (Griffithianas) que describen el aguzamiento, el tamaño y la posición de las fisuras. Cuando el sistema se encuentra en equilibrio desde el punto de vista de la mecánica clásica, las únicas coordenadas que varían son las coordenadas-G, cuyos cambios son denominados variaciones- $G$ y dados por $\delta a_{j}$.

Los estados del sistema cuerpo fisurado-cargas pueden ser clasificados con respecto al equilibrio y a la estabilidad. Los estados en los que el trabajo virtual es negativo para toda $\delta a_{j}>0$, son llamados estados de sub-equilibrio; los estados en los que existen algunas variaciones-G en las que el trabajo virtual es cero y en las restantes es negativo, son llamados estados de equilibrio; si por lo menos para una de las variaciones-G el trabajo virtual es positivo, son llamados estados de no-equilibrio.

Las condiciones de estabilidad también pueden ser expresadas en términos del trabajo virtual: los estados de sub-equilibrio evidentemente son estables, pues, no existen fuentes de energía dentro del 
sistema para cambiar el estado del sistema para cualquier estado vecino; los estados de no-equilibrio no pueden ser percibidos como unidades de equilibrio y por lo tanto, son inestables. Los estados de equilibrio pueden ser estables, neutros o inestables, siendo que el estado de equilibrio estable es el más importante en la teoría de la fractura y fatiga, pues es el caso típico de la propagación lenta y estable de la fisura. Una fisura propagará de forma estable cuando para alguna coordenada-G, $\delta_{G} W$ $=0$ y $\delta_{G}\left(\delta_{G} W\right)<0$ y cuando la condición $\delta_{G} W<0$ sea satisfecha para las otras.

En términos de fuerzas generalizadas, el trabajo virtual de las variaciones-G es representado por:

$\delta_{G} W=\sum_{j=1}^{m} G_{j} \delta a_{j}-\sum_{j=1}^{m} \Gamma_{j} \delta a_{j}$

donde las fuerzas generalizadas de propagación y de resistencia son $G_{j}$ y $\Gamma_{j}$ respectivamente. En términos de esas fuerzas, la fisura no crecerá cuando la condición $G_{j}<\Gamma_{j}$ sea cumplida. El inicio de su crecimiento se dará cuando $G_{j}=\Gamma_{j}$, que se tornará inestable cuando para alguna $\delta a_{k}, G_{k}>\Gamma_{k}$, lo que provocará la fractura total del componente.

\section{Movimiento del agente ambiental a través de una fisura}

El contenido de un agente ambiental activo en la vecindad de la punta de una fisura se caracteriza con una variable escalar que depende de un tiempo físico (o de un número de ciclos) y es denotada por $\mathrm{c}(\mathrm{t})$ o $\mathrm{c}(\mathrm{N})$. En casos simples, como en el caso de la solución de una sustancia activa simple, ese contenido es precisamente la concentración de esa solución y cuando el transporte del agente ocurre monótonamente, son aceptados modelos quasi-estacionarios. Uno de esos modelos es dado por:

$\frac{d c_{t}}{d t}=\frac{c_{h}-c_{t}}{T_{D}}+\frac{c_{b}-c_{t}}{\lambda_{D}} \frac{d a}{d t}$

donde el primer término del lado derecho representa el mecanismo de difusión y el segundo, el efecto de la propagación de la fisura. Los parámetros de gobierno que entran en esa ecuación son el tiempo característico de difusión, $T_{D}$, y la longitud característica de la zona de difusión, $\lambda_{D} \approx D / k_{0}$; $D$ es el coeficiente de difusión y $\mathrm{k}_{0}$ es un parámetro de transferencia de masa. $c_{t}$ es la concentración en la punta de la fisura, que generalmente no es la misma que la de su entrada, $\mathrm{c}_{\mathrm{e}}$, debido al movimiento del agente a través de ella. El parámetro $c_{b}$ es una concentración característica para el rápido crecimiento de la fisura; el parámetro $c_{h}$, que caracteriza una concentración estacionaria alcanzada en un tiempo $t>T_{D}$ y que depende de la concentración de entrada y de la longitud de la fisura, es dado por:

$c_{h}=c_{e}\left(1+\frac{a}{a_{\infty}}\right)^{-n_{a}}$

donde $a_{\infty}>0$ y $n_{a} \geq 0$ son constantes que dependen de las propiedades del material y del ambiente.

\section{FORMULACIÓN NUMÉRICA}

El propósito de la formulación de este problema es el de evaluar el caso de la propagación en régimen elástico, de una fisura lateral presente en un cuerpo material en estado plano de deformación y solicitada en el modo de fractura I por una tensión de tracción $\sigma_{\infty}$, utilizando el modelo matemático de Bolotin y Shipkov (2001a). La evolución de las variables radio y longitud de la fisura, daño y de su efecto sobre las fuerzas generalizadas de resistencia, forman un sistema de ecuaciones diferenciales de $1^{\text {er }}$ orden que es resuelto a través del método de Runge-Kutta de $4^{\circ}$ orden. El Factor de Intensidad de tensiones en las proximidades de la punta de una fisura de longitud a es calculado a través de la siguiente ecuación: 
$K_{\mathrm{I}}=\mathrm{Y} \sigma_{\infty} \sqrt{\pi \mathrm{a}}$

La expresión para el factor de forma $\mathrm{Y}$, empleado en (11), puede ser encontrada en el libro de Bolotin (1999).

Para evaluar el daño mecánico, los campos de tensión-deformación y de concentración en el cuerpo durante todo el proceso considerado deben ser conocidos, pudiendo ser utilizadas algunas aproximaciones simples, basadas en una analogía entre el factor concentrador de tensiones, $\mathrm{K}_{\mathrm{t}} \mathrm{y}$ el factor de intensidad de tensiones, $\mathrm{K}_{\mathrm{I}}$. Para una fisura plana en el modo de fractura I, esta analogía proporciona una fórmula aproximada para el factor de concentración de tensión en la punta y para la distribución de la tensión normal en su frente, que pueden ser calculados por:

$$
\begin{aligned}
& K_{t}=1+2 Y\left(\frac{a}{\rho}\right)^{1 / 2} \\
& \sigma=K_{t} \sigma_{\infty}\left[1+\frac{4(x-a)}{\rho}\right]^{-1 / 2}
\end{aligned}
$$

La Ecuación (12) es una extensión de la conocida fórmula de Neuber, que calcula el factor concentrador de tensiones llevando en consideración el factor geométrico Y. La Ecuación (13) es la más simple de las ecuaciones empíricas de Shin, donde $(x-a)=\xi=10 \rho$. Esta aproximación para la coordenada medida al frente de la punta de la fisura proporciona buenos resultados en problemas planos, pues esa distancia usualmente es suficiente para estimar el campo del daño, especialmente cuando los componentes distantes pueden ser despreciados (Bolotin, 1999).

Para considerar la acumulación del daño y la propagación de la fisura sometida a una carga estática y a una acción ambiental, se simula que su superficie está en contacto con un ambiente hidrogenado y que la concentración en su entrada, $c_{e}$, es constante. Se asume también que el material es elástico-lineal en toda la estructura y que la longitud de la fisura es apenas una coordenada-G. Bajo esas premisas, la tasa de liberación de energía de deformación elástica en el modo de fractura I, que corresponde a la fuerza generalizada de propagación, puede ser calculada por:

$\mathrm{G}_{\mathrm{I}}=\frac{\mathrm{K}_{\mathrm{I}}^{2}}{\mathrm{E}}\left(1-\mathrm{u}^{2}\right)$

donde E y u son el módulo de Young y el coeficiente de Poisson, respectivamente.

\section{RESULTADOS Y DISCUSIÓN}

Para la evaluación de la aplicabilidad del modelo, fue seleccionado un cuerpo material de acero de alta resistencia clase MARAGING T-250, con dimensiones $I=120 \mathrm{~mm}, w=80 \mathrm{~mm}, b=20 \mathrm{~mm}, a=8 \mathrm{~mm}$ y $\rho=50 \mu \mathrm{m}$, cuya geometría está mostrada en la Fig. 1(a). En el detalle de la Fig. 1(b) se muestran las zonas de proceso del daño provocado por la carga estática y por el hidrógeno. Las propiedades físicas y mecánicas del material adoptado están mostradas en la Tabla 1.

El problema de la propagación de la fisura fue resuelto mediante la solución del sistema formado por las ecuaciones de acumulación de daño, de variación del radio efectivo de la punta de la fisura, de transporte de masa y del efecto del daño sobre las fuerzas de resistencia a la propagación, fuertemente acopladas, mediante la aplicación del método de Runge-Kutta de $4^{\circ}$ orden. Los parámetros materiales adoptados para la simulación del problema están mostrados en la Tabla 2. 


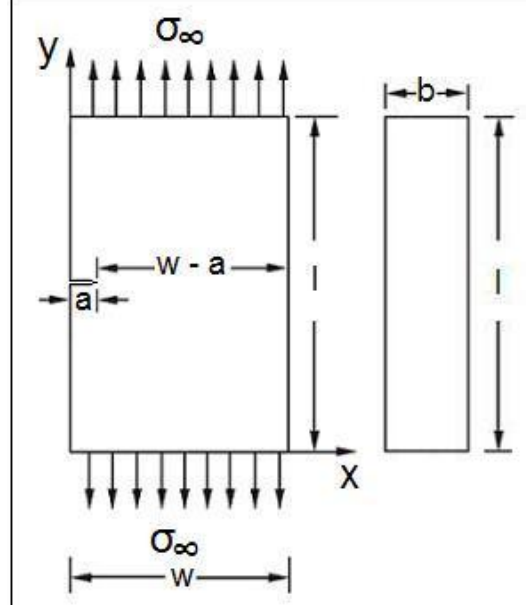

(a)

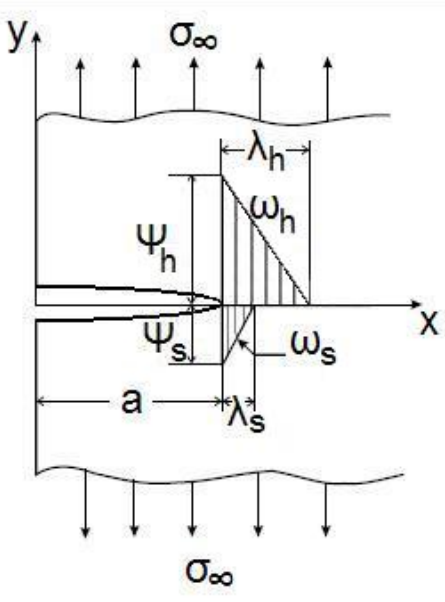

(b)

Fig. 1: (a) Cuerpo material con una fisura lateral bajo la tensión aplicada $\sigma_{\infty}$; (b) distribución de los daños al frente de la punta de la fisura (adaptado de Bolotin y Shipkov, 2001a).

Tabla 1: Propiedades físicas y mecánicas del acero MARAGING T-250 (Fuente: ALLVAC, 2000).

\begin{tabular}{|c|c|c|c|c|}
\hline $\begin{array}{c}\sigma_{r} \\
(\mathrm{~Pa})\end{array}$ & $\begin{array}{c}\sigma_{\mathrm{y}} \\
(\mathrm{Pa})\end{array}$ & $\begin{array}{c}\mathrm{E} \\
(\mathrm{Pa})\end{array}$ & $\begin{array}{c}\mathrm{U} \\
(\text { adim. })\end{array}$ & $\begin{array}{c}\mathrm{K}_{\mathrm{IC}} \\
(\mathrm{Pa} \cdot \sqrt{ } \mathrm{m})\end{array}$ \\
\hline $1,79 \mathrm{E}+09$ & $1,76 \mathrm{E}+09$ & $1,86 \mathrm{E}+11$ & 0,30 & $9,81 \mathrm{E}+07$ \\
\hline
\end{tabular}

Tabla 2: Parámetros materiales (Fuente: Bolotin, 1999).

\begin{tabular}{c|c|c|c|c|c|c|c|c|c|}
\hline $\begin{array}{c}\mathrm{C}_{\mathrm{b}} \\
(\operatorname{adim})\end{array}$ & $\begin{array}{c}\mathrm{T}_{\mathrm{D}} \\
(\mathrm{s})\end{array}$ & $\begin{array}{c}\mathrm{T}_{\mathrm{c}} \\
(\mathrm{s})\end{array}$ & $\begin{array}{c}\sigma_{\mathrm{s}} \\
(\mathrm{Pa})\end{array}$ & $\begin{array}{c}\sigma_{\mathrm{th}} \\
(\mathrm{Pa})\end{array}$ & $\begin{array}{c}\rho_{\mathrm{s}} \\
(\mu \mathrm{m})\end{array}$ & $\begin{array}{c}\rho_{\mathrm{b}} \\
(\mu \mathrm{m})\end{array}$ & $\begin{array}{c}\lambda_{\mathrm{D}} \\
(\mathrm{mm})\end{array}$ \\
\hline 0,5 & 100,0 & $5 \cdot \mathrm{E} 3$ & $5 \cdot \mathrm{E} 9$ & $250 \cdot \mathrm{E} 6$ & 10,0 & 100,0 & 1,00 \\
\hline $\begin{array}{c}\lambda_{\mathrm{a}} \\
(\mu \mathrm{m})\end{array}$ & $\begin{array}{c}\lambda_{\mathrm{h}} \\
(\mu \mathrm{m})\end{array}$ & $\begin{array}{c}\lambda_{\mathrm{s}} \\
(\mu \mathrm{m})\end{array}$ & $\begin{array}{c}\mathrm{X} \\
(\operatorname{adim})\end{array}$ & $\begin{array}{c}\mathrm{Y}_{0} \\
\left(\mathrm{~kJ} / \mathrm{m}^{2}\right)\end{array}$ & $\begin{array}{c}\mathrm{m}_{\mathrm{s}} \\
(\operatorname{adim})\end{array}$ & $\begin{array}{c}\Psi_{\mathrm{h}}^{\infty} \\
(\operatorname{adim})\end{array}$ & $\begin{array}{c}\mathrm{D} \\
\left(\mathrm{m}^{2} / \mathrm{s}\right)\end{array}$ & $\begin{array}{c}\mathrm{k}_{0} \\
(\mathrm{~m} / \mathrm{s})\end{array}$ \\
\hline 100,0 & 10,0 & 100,0 & 1,0 & 47,1 & 4,0 & 0,5 & $1 \cdot \mathrm{E}-8$ & $1 \cdot \mathrm{E}-5$ \\
\hline
\end{tabular}

Los resultados obtenidos luego de la aplicación del modelo son mostrados en las figuras 2 a la 5 .

La Fig. 2(a) muestra dos curvas que describen las soluciones numéricas del problema de transporte de masa a la punta de una fisura, dado por la Ecuación 9, para las condiciones iniciales $c_{t}(0)=0$ y $c_{t}(0)=1$. En la obtención de esas soluciones fue asumido que la fisura propaga con una tasa constante, despreciándose la absorción en sus caras. Las dos curvas tienden a convergir rápidamente para una concentración estacionaria, $c_{h}$, siendo el tiempo característico para esa convergencia, de aproximadamente 500s. Como era de esperarse, la concentración estacionaria es alcanzada con mayor rapidez cuando $c_{t}(0)=1$, dada la facilidad para la difusión proporcionada por la generación de un gradiente de concentraciones.

La Fig. 2(b) muestra la evolución de la concentración de hidrógeno en la punta de la fisura luego de la aplicación del modelo completo, que incluye transferencia de masa, acumulación de daño y crecimiento de la fisura. Fue asumido daño inicial y concentración de entrada nulos. La tensión aplicada, $\sigma_{\infty}=150 \mathrm{MPa}$, fue mantenida constante durante toda la evolución de la fisura. 
En la curva generada se puede observar que el transporte del agente en la etapa inicial del proceso es bastante rápido, respondiendo a la velocidad de difusión del hidrógeno en el material. La segunda etapa, donde es alcanzada una concentración estable, corresponde a un valor crítico de esa concentración y al inicio de la propagación de la fisura. Cuando ella se torna más intensa, el transporte de hidrógeno para la punta de fisura es dificultado, lo que provoca la disminución de su concentración, observada en la declinación de la curva en la tercera etapa.

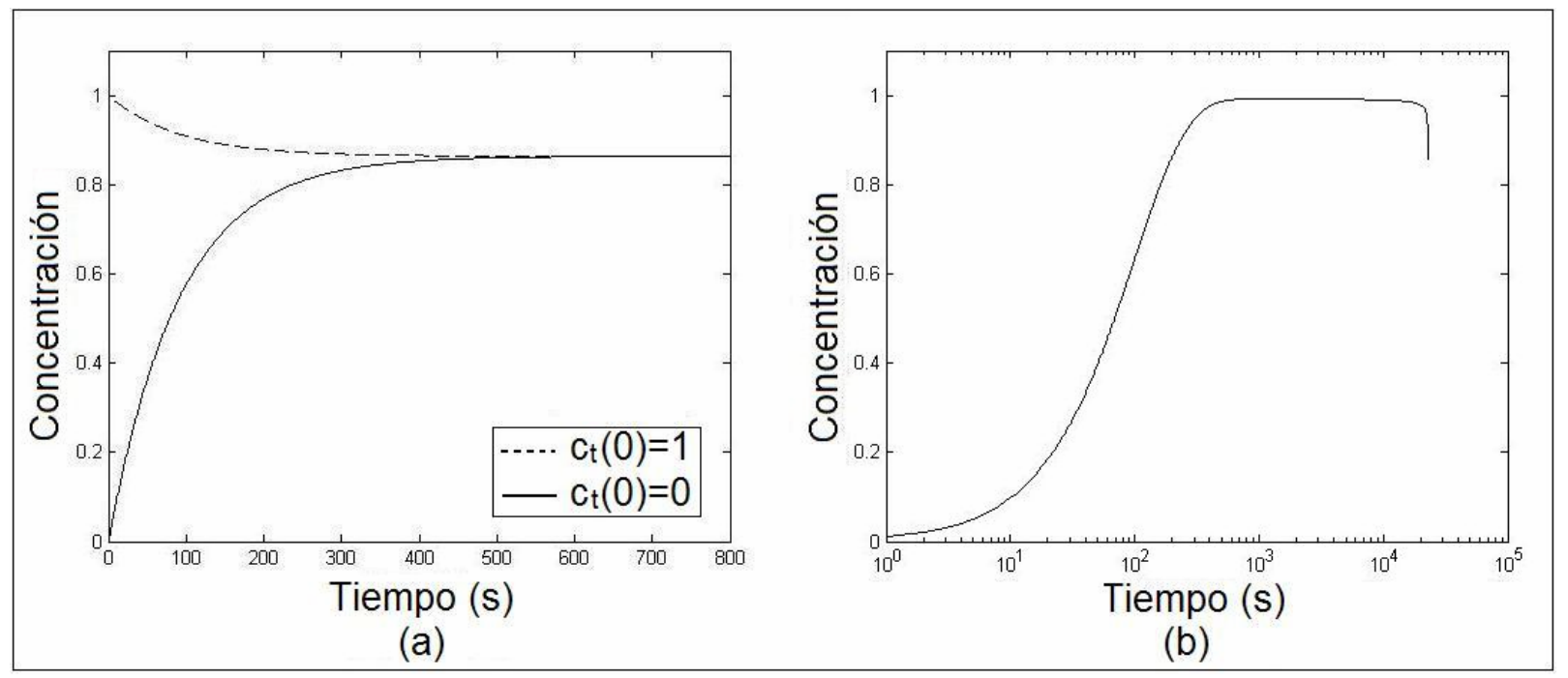

Fig. 2: Evolución de la concentración hidrógeno en la punta de la fisura: (a) solución de la ecuación de transporte de masa; (b) aplicación del modelo completo para $\sigma_{\infty}=150 \mathrm{MPa}$.

La Fig. 3(a) presenta tres curvas que muestran la evolución del radio efectivo de la punta de la fisura para diferentes concentraciones de entrada, $c_{e}$, normalizadas con $c_{h}$ e ilustran fenómenos que son difíciles de ser observados directamente:

La parte creciente de cada curva representa el embotamiento de la punta de la fisura debido al proceso de acumulación de daño, que es muy rápido, condicionado por la rápida difusión del hidrógeno. Cuando finaliza el proceso de embotamiento se inicia el crecimiento de la fisura, momento correspondiente al inicio del aguzamiento de su punta, que es acelerado en la medida en que la concentración de hidrógeno aumenta.

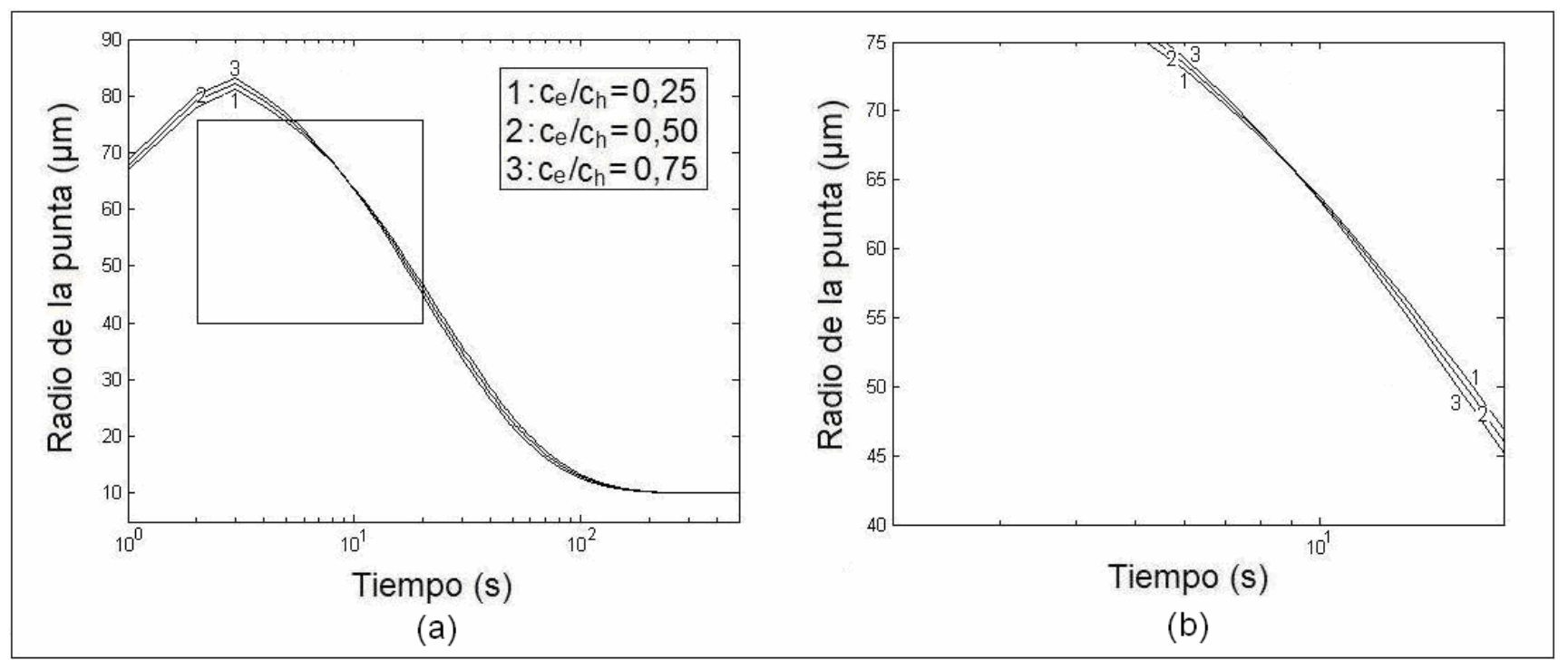

Fig. 3: (a) evolución del radio efectivo de la punta de la fisura para una carga de $200 \mathrm{MPa}$ y diferentes concentraciones normalizadas de $c_{e} / c_{h} ;(b)$ ampliación del área destacada. 
El detalle de la Fig. 3(b) permite observar ese fenómeno: a mayores concentraciones de hidrógeno ocurre un embotamiento más acentuado debido a la degradación provocada por el efecto combinado del cargamento mecánico y principalmente, del agente ambiental; cuando ocurre el aguzamiento de la fisura, el tiempo de inicio para su crecimiento disminuye, nuevamente, con el aumento de la concentración del hidrógeno. Ambos hechos son consistentes con observaciones macroscópicas del fenómeno de fragilización por hidrógeno.

En las Figs. 4(a) y 4(b) se muestra la evolución del crecimiento de la fisura, que se encuentra condicionada a la acción de la carga estática y del hidrógeno, en distintas condiciones de aplicación de la carga y de concentración de ese agente agresivo. En ellas se observa que la evolución inicial es muy lenta y que casi no sufre alteraciones en el tiempo; solamente a partir de transcurrido un cierto tiempo la fisura presenta un crecimiento cada vez más rápido, provocado por el aumento de las fuerzas de propagación y la disminución de las fuerzas de resistencia.

Bajo determinadas condiciones de carga y concentración, el punto más alto de las curvas coincide con la dimensión crítica de la fisura, $\mathrm{a}_{\mathrm{c}}$, representando la fractura instantánea del componente debido a la inestabilidad provocada cuando las fuerzas de resistencia a la propagación son superadas. En niveles muy bajos de la carga aplicada, representa la falla de la estructura provocada simplemente por el crecimiento de la fisura hasta el límite de las dimensiones del cuerpo material, debido a la ausencia del ligamento necesario para que la dimensión crítica de la fisura sea alcanzada.

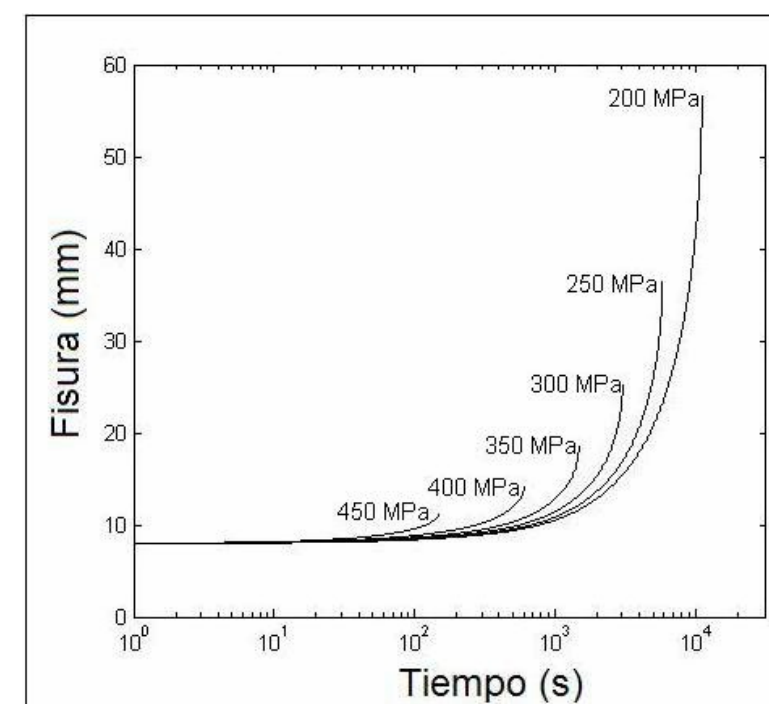

(a)

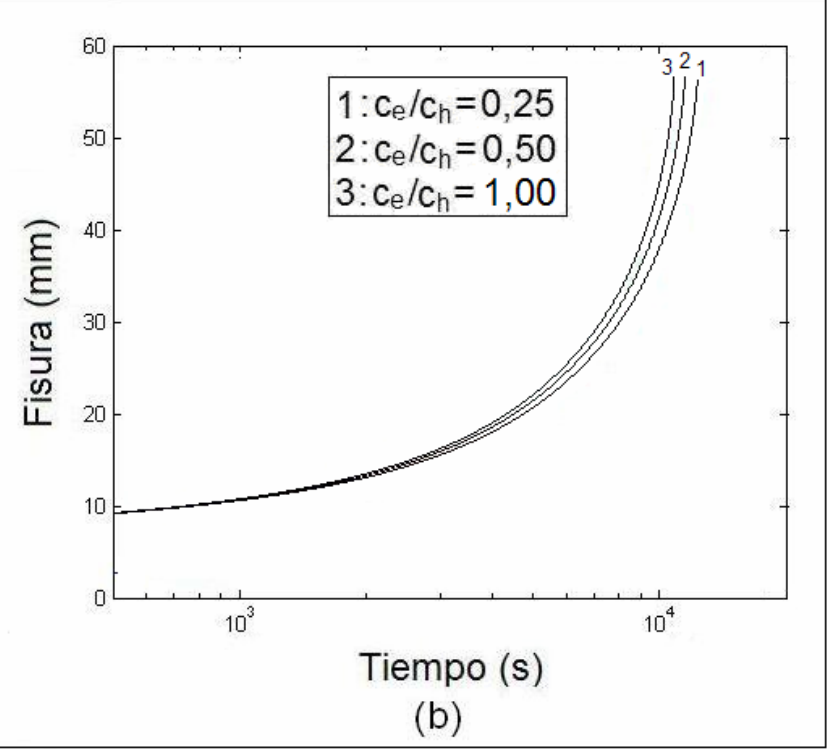

(b)

Fig. 4: Evolución del crecimiento de la fisura (a) para diferentes cargas aplicadas y una concentración normalizada de $c_{e} / c_{h}=1,00$; (b) para una carga de $200 \mathrm{MPa}$ y diferentes concentraciones de $c_{e} / c_{h}$.

Las curvas de la Fig. 4(a) muestran esa evolución para diferentes cargas aplicadas y la misma concentración del agente $\left(c_{e} / c_{h}=1,00\right)$. Se observa que, en la medida que la solicitación aumenta, los tiempos de fractura y las dimensiones críticas de la fisura disminuyen. Las curvas de la Fig. 4(b), levantadas para la misma carga aplicada y diferentes concentraciones de hidrógeno, muestran una disminución en el tiempo de inicio de propagación de la fisura y de la fractura final en la medida en que la concentración aumenta. Estos resultados también son consistentes con observaciones experimentales del fenómeno.

En la Fig. 5 se muestra la evolución del factor de intensidad de tensiones con el tiempo, provocada por el crecimiento de la fisura para diferentes magnitudes de carga y una misma concentración de hidrógeno. La tendencia de comportamiento de ellas es la de crecer hasta alcanzar el valor de la tenacidad a la fractura del material (representada por la línea horizontal), momento en que ocurrirá el colapso de la estructura. 
Esa tendencia es consistente con la de curvas obtenidas a partir de resultados experimentales (Hertzberg, 1996) y permite visualizar el proceso de propagación de fisuras sub-críticas cuando se encuentran sometidas a una carga mecánica estática y a una acción ambiental, en este caso, caracterizada por la difusión del hidrógeno a través de la estructura cristalina del material.

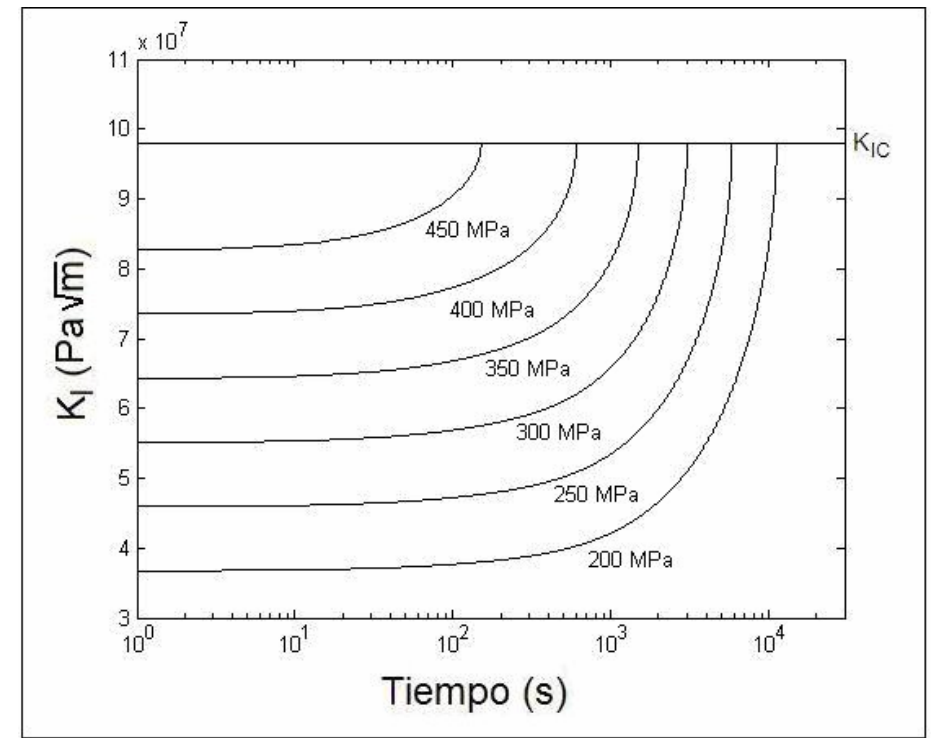

Fig. 5: Evolución del factor de intensidad de tensiones para diferentes cargas aplicadas y una concentración normalizada de $\mathrm{c}_{\mathrm{e}} / \mathrm{c}_{\mathrm{h}}=1,00$.

\section{CONCLUSIONES}

El modelo de Mecánica del Daño adoptado muestra buena flexibilidad para su acoplamiento con modelos estudiados en la Mecánica de la Fractura, dado que también utiliza parámetros empleados por ella, tales como la tasa de energía de deformación elástica, el factor de intensidad de tensiones y el radio efectivo de la punta de la fisura. Los resultados obtenidos mostraron buena correlación y consistencia con los fenómenos estudiados, permitiendo un mejor entendimiento del proceso de inicio y propagación de fisuras en ambientes agresivos.

El comportamiento de la curva de evolución del factor de intensidad de tensiones con el tiempo, obtenida para diferentes condiciones de carga, muestra un comportamiento similar al encontrado en la literatura. Esos resultados demuestran la validez del modelo adoptado para la simulación del inicio y crecimiento de fisuras en régimen elástico.

La simulación del crecimiento de una fisura, como resultado del proceso de deterioro del material debido a la aplicación de la carga estática y a la acción del hidrógeno, mostró que el tiempo de inicio y de crecimiento de fisuras disminuye cuando la concentración del agente ambiental aumenta, lo que es consistente con observaciones macroscópicas del fenómeno de la fragilización por hidrógeno.

\section{AGRADECIMIENTOS}

Los autores agradecen a los órganos FINEP, CTPETRO, CNPq, PETROBRAS, RPCMod y al programa ANP/UFCG/PRH-25 por haber financiado este trabajo de investigación.

\section{NOMENCLATURA}

a Longitud de la fisura $(\mathrm{mm})$

$\mathrm{a}_{\mathrm{c}} \quad$ Dimensión crítica de la fisura $(\mathrm{mm})$

b Espesura del cuerpo material $(\mathrm{mm})$

$\mathrm{c}_{\mathrm{b}} \quad$ Concentración característica para el rápido crecimiento de la fisura (ppm) 
$\mathrm{C}_{\mathrm{e}} \quad$ Concentración de entrada en la boca de la fisura (ppm)

$\mathrm{C}_{\mathrm{h}} \quad$ Concentración estacionaria para una fisura estacionaria (ppm)

$\mathrm{C}_{\mathrm{t}} \quad$ Concentración en la punta de la fisura (ppm)

D Constante de difusión $\left(\mathrm{m}^{2} / \mathrm{s}\right)$

E Módulo de Young $(\mathrm{Pa})$

$\mathrm{G}_{\mathrm{j}} \quad$ Fuerza generalizada de propagación $\left(\mathrm{J} / \mathrm{m}^{2}\right)$

G Tasa de liberación de energía de deformación elástica en el modo de fractura I $\left(\mathrm{J} / \mathrm{m}^{2}\right)$

$\mathrm{k}_{0} \quad$ Parámetro de transferencia de masa $(\mathrm{m} / \mathrm{s})$

$\mathrm{K}_{\mathrm{I}} \quad$ Factor de intensidad de tensiones en el modo de fractura I ( $\left.\mathrm{Pa} \sqrt{\mathrm{m}}\right)$

$\mathrm{K}_{\mathrm{IC}} \quad$ Factor de intensidad de tensiones crítico en el modo de fractura I en EPD (Pa $\left.\sqrt{\mathrm{m}}\right)$

$\mathrm{K}_{\mathrm{t}} \quad$ Factor concentrador de tensiones (adim.)

I Longitud del cuerpo material $(\mathrm{mm})$

$\mathrm{m}_{\mathrm{s}} \quad$ Exponente de la ecuación de daño disperso debido a la carga estática (adim.)

$t_{c} \quad$ Constante del tiempo (s)

w Ancho del cuerpo material $(\mathrm{mm})$

Y Factor de forma (adim.)

a Parámetro material (adim.)

Y Trabajo específico de fractura $\left(\mathrm{J} / \mathrm{m}^{2}\right)$

Yo Trabajo específico de fractura para un cuerpo no damnificado $\left(\mathrm{J} / \mathrm{m}^{2}\right)$

$\Gamma_{\mathrm{j}} \quad$ Fuerza generalizada de resistencia $\left(\mathrm{J} / \mathrm{m}^{2}\right)$

$\delta a_{i} \quad$ Variaciones-G $(\mu \mathrm{m})$

$\delta W_{G} \quad$ Trabajo virtual de las variaciones-G $(\mathrm{J})$

$\lambda_{a} \quad$ Distancia de recorrido de la punta para obtener un aguzamiento representativo $(\mu \mathrm{m})$

$\lambda_{\mathrm{D}} \quad$ Longitud característica de la zona de difusión $(\mu \mathrm{m})$

$\lambda_{\mathrm{h}} \quad$ Longitud de la zona de proceso de la fragilización $(\mu \mathrm{m})$

$\lambda_{\mathrm{s}} \quad$ Longitud de la zona de proceso del daño debido a la carga mecánica $(\mu \mathrm{m})$

$\rho \quad$ Radio de curvatura de la punta de la fisura $(\mu \mathrm{m})$

$\rho_{\mathrm{s}} \quad$ Radio de curvatura de la punta aguzada $(\mu \mathrm{m})$

$\rho_{\mathrm{b}} \quad$ Radio de curvatura de la punta embotada $(\mu \mathrm{m})$

$\sigma_{\infty} \quad$ Tensión nominal $(\mathrm{Pa})$

$\sigma_{\mathrm{r}} \quad$ Tensión de resistencia máxima $(\mathrm{Pa})$

$\sigma_{\mathrm{s}} \quad$ Parámetro de resistencia al daño por la carga estática $(\mathrm{Pa})$

$\sigma_{\text {th }} \quad$ Tensión umbral $(\mathrm{Pa})$

$\sigma_{y} \quad$ Tensión de fluencia $(\mathrm{Pa})$

$\mathrm{T}_{\mathrm{D}} \quad$ Tiempo característico de difusión (s)

u Módulo de Poisson (adim.)

X Parámetro que caracteriza la tenacidad a la fractura residual (adim.)

$\Psi_{\mathrm{h}} \quad$ Daño en la punta de la fisura debido a la fragilización por hidrógeno (adim.)

$\psi_{\mathrm{s}} \quad$ Daño en la punta de la fisura debido a la carga estática (adim.)

$\Psi_{\mathrm{h}}^{\infty} \quad$ Daño en la punta de la fisura correspondiente a un estado de saturación (adim.)

$\omega_{\mathrm{h}} \quad$ Daño disperso debido a la fragilización por hidrógeno (adim.)

$\omega_{\mathrm{s}} \quad$ Daño disperso debido a la carga estática (adim.)

\section{REFERENCIAS}

ALLVAC, Technical Data Sheet-Vascomax Nickel Maraging Alloys, (en línea), (2000). http//www.allvac.com. Acceso: 20 de Marzo de 2007.

Barbosa, J.M.A.; Análise do Acoplamento Termomecânico em Barras Elasto-viscoplásticas com Dano, Tesis de Doctorado, Depto. de Ing. Mecánica, PUC - Rio, Rio de Janeiro, Brasil (1998).

Bolotin, V.V.; Mechanics of Fatigue, CRC Press, Boca Ratón, EUA (1999).

Bolotin, V.V. y A.A. Shipkov; Mechanical Aspects of Corrosion Fatigue and Stress Corrosion Cracking, International Journal of Solids and Structures: 38, 7297-7318 (2001a). 
Bolotin, V.V. y A.A. Shipkov; Prediction of the Growth of Fatigue Cracks Taking Environmental Factors into Account, J. Appl. Maths. Mechs.: 65(6), 1001-1010 (2001b).

Celentano, D. y J.L. Chaboche; Experimental and Numerical Characterization of Damage Evolution in Steels, Int. J. Plasticity: 23, 1739-1762 (2007).

Duda, F.P., J.M.A. Barbosa, L.J. Guimarães y A.C. Souza; Modeling of Coupled DeformationDiffusion-Damage in Elastic Solids, International Journal of Modeling and Simulation for the Petroleum Industry: 1(1), 85-93 (2007a).

Duda, F.P., A.C. Souza, L.J. Guimarães y J.M.A. Barbosa; An One Dimensional Coupled Model for Deformation, Degradation and Solute Diffusion in Elastic Solids, Mech. of Solids in Brazil: 1, 199-213 (2007b).

Gangloff, R.P.; Hydrogen Assisted Cracking of High Strength Alloys, In Comprehensive Structural Integrity, Elsevier Science (6), 31-101, New York, EUA (2003).

Gangloff, R.P.; Critical Issues in Hydrogen Assisted Cracking of Structural Alloys, In Environment Induced Cracking of Metals, Elsevier Science, 21-43, Oxford, Reino Unido (2005).

Hertzberg, R.W.; Deformation and Fracture Mechanics of Engineering Materials, John Willey \& Sons, New York, EUA (1996).

Louthan Jr., M.R.; Hydrogen Embrittlement of Metals: A Primer for the Failure Analyst, J Fail. Anal. and Preven.: 8, 289-307 (2008).

Maimí, V.P., Modelización Constitutiva y Computacional del Daño y la Fractura en Materiales Compuestos, Tesis de Doctorado, Universitat de Girona, Girona, España (2006).

Palma Carrasco, J.A., Aplicação de Modelos de Fratura Elástica e de Dano para a Simulação Numérica da Influência do Hidrogênio na Propagação de Trincas em Aços de Alta Resistência, Tesis de Maestría, Depto. Eng. de Materiais, UFCG, Campina Grande, Brasil (2008).

Stroe, M.E.; Hydrogen Embrittlement of Ferrous Materials, Tesis de Ph.D., Faculty of Applied Sciences, Université Libre de Bruxelles, Bruselas, Bélgica (2006).

Villalba, E. y A. Atrens; Hydrogen Embrittlement and Rock Bolt Stress Corrosion Cracking, Engineering Failure Analysis: 16, 164-175 (2009).

Yongwon, L. y R.P. Gangloff; Measurement and Modeling of Hydrogen Environment Assisted Cracking of Ultra High-Strength Steel, Metall. and Mat. Trans. A: 38A, 2174-2190 (2007).

Zhang, Y.P. y otros seis autores; Hydrogen Assisted Cracking of Maraging T-250 Steel, Materials Science and Engineering A: 471, 34-37 (2007). 